Article

\title{
Structure of Manganese Oxide Nanoparticles Extracted via Pair Distribution Functions
}

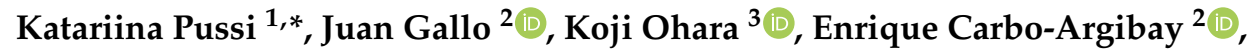 \\ Yury V. Kolen'ko ${ }^{2} \mathbb{D}$, Bernardo Barbiellini ${ }^{1,4}{ }^{-}$, Arun Bansil ${ }^{4}$ and Saeed Kamali $5,6, *$ \\ 1 School of Engineering Science, LUT University, FI-53851 Lappeenranta, Finland; Bernardo.Barbiellini@lut.fi \\ 2 International Iberian Nanotechnology Laboratory, 4715-330 Braga, Portugal; juan.gallo@inl.int (J.G.); \\ enrique.carbo@inl.int (E.C.-A.); yury.kolenko@inl.int (Y.V.K.) \\ 3 Japan Synchrotron Radiation Research Institute, SPring-8, 1-1-1 Kouto, Sayo-cho, Sayo-gun, Hyogo 679-5198, \\ Japan; ohara@spring8.or.jp \\ 4 Physics Department, Northeastern University, Boston, MA 02115, USA; ar.bansil@northeastern.edu \\ 5 Mechanical, Aerospace and Biomedical Engineering Department, University of Tennessee Space Institute, \\ Tullahoma, TN 37388, USA \\ 6 Department of Physics and Astronomy, Middle Tennessee State University, Murfreesboro, TN 37132, USA \\ * Correspondence: katariina.pussi@lut.fi (K.P.); skamali@etsi.edu (S.K.)
}

Received: 27 December 2019; Accepted: 16 March 2020; Published: 18 March 2020

\begin{abstract}
The structure of nanoparticles has been difficult to determine accurately because the traditional structure methods rely on large monocrystals. Here, we discuss the structure of nanoparticles based on real-space modeling of the pair distribution function obtained by a Fourier transformation of the high-energy X-ray scattering structure factor. In particular, we consider X-ray scattering data taken from colloidal manganese oxide nanoparticles used in Lithium-ion batteries, air-purification, and biomedical systems, which are known to exist in various nanometer-sized polymorphs. Insight is thus obtained into characterizing the structural relaxation of the $\mathrm{MnO}_{6}$ octahedra, which are the key building blocks of oxide nanoparticles, important in many technologies.
\end{abstract}

Keywords: nanoparticles; high energy X-ray diffraction (HE-XRD); electrodes; Li-ion batteries; air purification; contrast agent; pair distribution function (PDF) analysis

\section{Introduction}

Among the metal oxides, manganese oxide plays a vital role in technological applications. Manganese oxide microstructures and nanostructures are used, for example, in waste-water treatment, catalysis, biomedicine, air-purification sensors, super-capacitors, and rechargeable batteries [1-8]. Transition metal oxides, especially $\mathrm{MnO}$ and $\mathrm{MnO}_{2}$, are good candidates for anodes in batteries as they provide high capacity and low cost and are also environmentally friendly [9-11].

X-ray scattering techniques are useful for characterizing the sizes of crystals and particles as well as their crystallographic phases, which taken together control their physical properties. These techniques are generally non-destructive and measure properties averaged over an ensemble of many particles. Most crystal structures have been determined via X-ray diffraction (XRD) experiments. The scattering pattern from a crystal typically consists of several tens or even hundreds of Bragg reflections from various atomic planes in the lattice. Crystal structures can be specified in terms of just a few variables, such as the unit cell parameters and the positions of the symmetry-independent atoms.

Direct imaging techniques such as electron microscopy also provide unique opportunities for structure analysis at a more local scale and thus complement diffraction techniques $[12,13]$. A transmission electron microscope (TEM), for example, can provide multidimensional analysis 
capabilities for characterizing nanoparticles and can be used to image nanoscale features with atomic resolution. Electron microscopy normally measures a two-dimensional (2D) projection of the three-dimensional (3D) structure, although the 3D structure can be obtained via electron tomography [14-16]. The increasing complexity of nanomaterials has driven the development of even more advanced characterization techniques combined with tomography. Energy-dispersive X-ray (EDS) spectroscopy [17,18] and electron energy loss spectroscopy (EELS) [19] are examples of such advances, which have enabled the investigation of the morphologies, compositions, and oxidation states of nanomaterials.

X-ray scattering of nanoparticles is much weaker than that of bulk materials due to their small volume and limited coherence. In order to increase the signal, samples must be measured either over long times or with high-flux sources. For nanostructures, diffraction patterns often show only a few Bragg reflections, which makes conventional crystallography challenging. Moreover, the assumptions underlying the analysis neglect information on structural disorder due to high frustrated surface contributions. The key is to recognize that a large amount of information here is stored in diffuse scattering, which is normally not taken into account in conventional X-ray analysis. It is clear that other methods are needed for a complete understanding of the structure of nanoparticles.

High-energy XRD (HE-XRD) measurements combined with a Pair Distribution Function (PDF) analysis can yield valuable information on the short-range atomic ordering of nanocrystalline materials with some degree of structural coherence and periodicity. A large body of literature [20-37] details how the PDF analysis can enable a complete structural investigation of a nanostructured material. Application to nanomaterials, however, has become practical only recently, with the advent of high-flux and high-energy synchrotron radiation sources. Our study is motivated by the growing interest in applying high-energy $X$-ray scattering techniques to investigate nanostructures.

We emphasize that the PDF method analyzes all the scattered intensity and it can therefore probe deviations from perfect crystallinity. This is important because nanomaterials can be ordered and possess periodic atomic arrangements like ordinary crystals, but they can also be disordered and/or have nonperiodic atomic arrangements like glasses. In the former case, as we have already noted, the crystal structure may be described in terms of a relatively small number of parameters that can be conveniently computed and predicted. In the latter case, however, the critical information is stored in the diffuse scattering pattern. The three-dimensional structure now needs to be described by a sufficiently large (statistically representative) number of atomic configurations and the related coordinates and chemical types of the various involved atoms. It has become possible to address this challenging problem in recent years, as greatly increased computational power has become available [25]. Our study addresses the characterization of nanostructures, which have not been investigated as extensively as the crystalline structures, despite their crucial role in current, emerging, and future technologies.

\section{Experimental Details}

MnO nanoparticles were obtained by adapting the protocol of Gallo et al. [38] as follows. First, a manganese (II) oleate complex was produced via an exchange chemical reaction between manganese (II) chloride and sodium oleate. A quantity of $1.24 \mathrm{gm}$ of the resulting manganese (II) oleate and $10 \mathrm{gm}$ of 1-hexadecene were then combined in a $50 \mathrm{~mL}$ round-bottom flask attached to a Schlenk line. The mixture was degassed at $80^{\circ} \mathrm{C}$ for $90 \mathrm{~min}$, and then the vacuum was switched to Ar atmosphere. Next, the flask was heated to $280{ }^{\circ} \mathrm{C}$ and held at this temperature for $30 \mathrm{~min}$, followed by cooling to room temperature. Nanoparticles were precipitated by adding $10 \mathrm{~mL}$ of acetone, collected by centrifugation at $9000 \mathrm{rpm}$ for $10 \mathrm{~min}$, re-dispersed in hexane, washed again with acetone and collected by centrifugation. Finally, the nanoparticles were re-dispersed in toluene to obtain a $10 \mathrm{mg} \cdot \mathrm{mL}^{-1} \mathrm{stock}$ of colloidal MnO nanoparticles.

Thin Si wafer substrates measuring $1 \mathrm{~cm} \times 1 \mathrm{~cm}$ were first cleaned using ultrasonication in acetone, ethanol, and isopropanol, and then dried by flashing compressed air. The stock dispersion of the 
nanoparticles in toluene was drop-casted onto a substrate, and, then, the solvent was allowed to evaporate slowly in ambient conditions.

Morphology of the synthesized nanoparticles was determined by transmission electron microscopy (TEM) using a JEOL JEM 2100 working at $200 \mathrm{kV}$ TEM. The average diameter of the nanoparticles was determined by measuring the diameters of 200 particles and fitting the histograms to a Gaussian distribution function. Figure 1 shows a high resolution (HR)-TEM image of the as-synthesized colloidal $\mathrm{MnO}$ nanoparticles. The TEM picture was taken before deposition on the silica substrate. As discussed below, the silica substrate seems to alter the crystallinity of the sample. Fast Fourier transform (FFT) images including plane-spacing calculations are shown in the Supplementary Material.

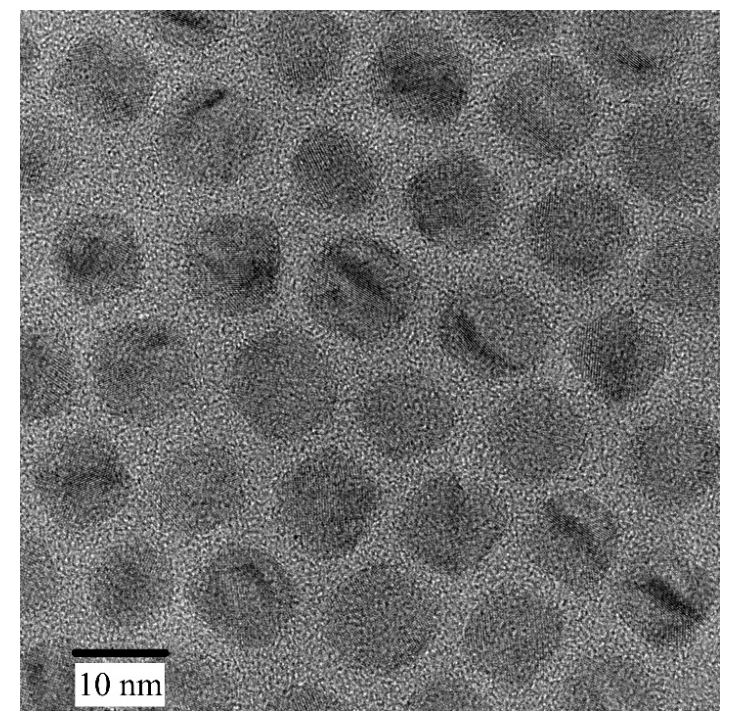

Figure 1. HR-TEM image of the colloidal MnO nanoparticles. The HR-TEM image was taken before deposition on the silica substrate.

HE-XRD measurements with a photon wavelength of $0.21 \AA$ were performed at beamline BL04B2 at the Japanese synchrotron facility SPring- 8 . An ionization chamber was used for monitoring the intensity of the incident $X$-rays. Three $\mathrm{CdTe}$ detectors were used to measure the intensity of the scattered $\mathrm{X}$-rays. The small-angle scattering part of the signal was measured with a two-axis diffractometer to cover the low-Q region $\left(\approx 0.1 \AA^{-1}\right)$. A vacuum chamber at HE-XRD setup was used to suppress air scattering around the sample. The HE-XRD setup at this beamline is described in more detail elsewhere [39]. Although radiation damage to individual nanoparticles should be reduced as much as possible, nanoparticles on a metallic substrate can be healed through a healing mechanism that involves a neutralization process [40].

Experimental $I(Q)$ data were obtained for $\mathrm{MnO}$ nanoparticles of approximately $10 \mathrm{~nm}$ size. Background intensity was determined by carrying out a measurement on a bare substrate. The proper subtraction of the background is important for capturing the diffuse scattering signal in the data properly. Figure 2 shows the experimental $I(Q)$ results for the $\mathrm{MnO}$ film sample. Only one Bragg-type peak, centered at $3.3 \AA^{-1}$, is seen. Other peaks are an order of magnitude weaker and represent diffuse scattering. It is likely that the diffraction peaks of the $\mathrm{MnO}$ film are too weak to be observed due to the strong and sharp diffraction peaks of the silicon substrate, as has been reported previously for $\mathrm{MnO}_{\mathrm{x}}$ on stainless steel [6]. TEM analysis provides grain size information. The particle size obtained via TEM is usually larger than the crystallite size given by XRD. This is to be expected since a particle typically consists of several crystallites. Information on crystallite size can be obtained by analyzing X-ray diffraction peaks where the peak width is inversely proportional to crystallite size, effects of other factors that contribute to the peak width such as instrumental resolution, temperature and microstrain notwithstanding. 


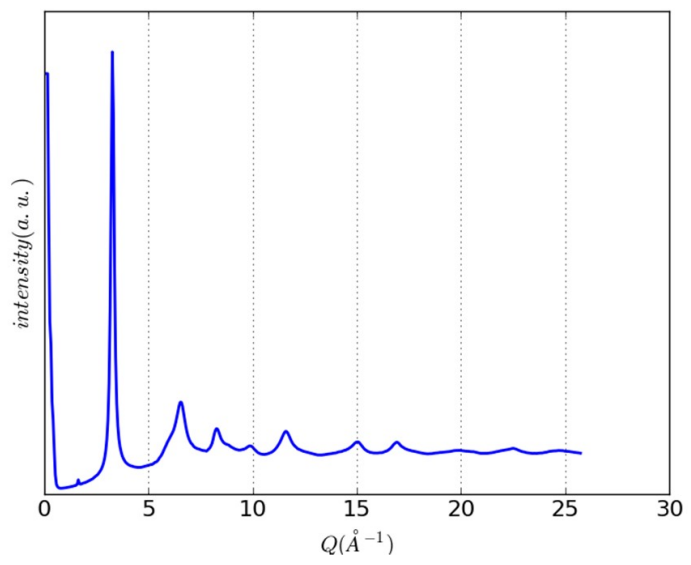

Figure 2. $I(Q)$ data for the $\mathrm{MnO}$ nanoparticle film sample.

\section{Results and Discussion}

\subsection{Computational Details}

Note that the intensity of diffuse scattering, which is related to short and medium range order, is orders of magnitude smaller than that of the Bragg peaks. Fourier transformation of experimental total scattering data gives the pair distribution function, which is a direct probe of interatomic distances in real space. The window in $Q$-space, which depends on the radiation source, defines the accuracy of the data in the real space. For elastic scattering, the diffraction vector, $Q$, has a magnitude

$$
|Q|=\frac{4 \pi \sin (\theta)}{\lambda}
$$

where $\lambda$ is the wavelength of incident photons and $2 \theta$ is the scattering angle. Since $\sin \theta \leq 1$, the experimentally accessible range of $Q$ is limited to less than $4 \pi / \lambda$. For example, the $\mathrm{CuK}_{\alpha}$ radiation, which is a common radiation source, has a wavelength of $1.54 \AA$ with a $Q$-range of about $8 \AA^{-1}$, but for investigating nanoparticles, a $Q_{\max }$ larger than $20 \AA^{-1}$ is needed. For our $\mathrm{MnO}$ data here, we have $Q_{\max }=25 \AA^{-1}$, which enables us to achieve relatively good real-space accuracy.

We will use the PDF approach as a 'small-box modelling', where one considers relatively small unit cells with periodic boundary conditions. For periodic model structures, the PDF, $G(r)$, can be computed in real space as:

$$
G(r)=\frac{1}{N r\langle b\rangle^{2}} \sum b_{i} b_{j} \delta\left(r-r_{i j}\right)-4 \pi r \rho_{0}
$$

where $b_{i, j}$ are related to thermal movements of the scattering atoms, $r_{i j}$ are the relative positions of the scattering atoms, $N$ is the number of particles and $\rho_{0}$ is the particle density.

The PDF provides atomic-scale structural insights from the distribution of atom-atom distances ranging from the local coordination scale to several nanometers. In addition to atom-pair separations (peak positions), the PDF also provides direct information about coordination number (integrated peak intensity), static and dynamic disorder (peak shape), and the coherent scattering-domain size (peak attenuation). Modelling the PDF for known periodic structures allows us to assess the effects of modifying the lattice constant, thermal vibration parameters, atomic positions and site occupancies. In addition, PDF-dependent shape parameters can also be used for extracting the radius of a spherically shaped nanoparticle.

\subsection{An Illustrative Analysis of the MnO Data}

Manganese can exist in the form of a variety of stable oxides $\left(\mathrm{MnO}, \mathrm{Mn}_{3} \mathrm{O}_{4}, \mathrm{Mn}_{2} \mathrm{O}_{3}, \mathrm{MnO}_{2}\right)[41,42]$, which crystallize in different types of structures. Associated with this wide diversity of crystal 
forms, defect chemistry, morphology, porosity and textures, manganese oxides exhibit a variety of distinct electrochemical properties. For example, $\mathrm{MnO}_{2}$ exists in six different polymorphs (pyrolusite, ramsdellite, hollandite, intergrowth, spinel, and layered), all of which share basic structural features-small $\mathrm{Mn}^{4+}$ ions in a spin-polarized $3 \mathrm{~d}^{3}$ configuration and large, highly polarizable $\mathrm{O}^{2-}$ ions in a spin-unpolarized $2 \mathrm{p}^{6}$ configuration, which are arranged in corner- and edge-sharing $\mathrm{MnO}_{6}$ octahedra. These octahedral units are characteristic for each oxidation state of manganese oxide and play a crucial role in determining the electrochemical properties of various oxides.

$\mathrm{MnO}$ crystallizes in the so-called rock-salt structure, which is a face-centered cubic (fcc) lattice with a 6:6 octahedral coordination. The experimental lattice constant at room temperature is $a=4.444 \AA$ [43]. The first three coordination shells for Mn-O distances are $(1 / 2) a=2.22 \AA,(\sqrt{ } 3 / 2) a=3.84 \AA$, and $(\sqrt{5} / 2) a$ $=4.97 \AA$, and the corresponding Mn-Mn (or O-O) distances are $(\sqrt{ } 2 / 2) a=3.14 \AA, a=4.44 \AA$ and $(\sqrt{ }(3 / 2)) a=5.44 \AA$.

We obtained the PDF by using PDFgetX3 [44], which is a command-line utility for extracting atomic pair distribution functions from X-ray diffraction data. Data up to $Q=25.00 \AA^{-1}$ were used using the Fourier transform, giving a real-space resolution of $\Delta r \approx 0.25 \AA$. The PDF data were further analyzed using the DiffPy-CMI package [45], which is a library of Python modules for robust modelling of nanostructures in crystals, nanomaterials, and amorphous materials.

Figure 3 shows the PDF for MnO obtained by converting the experimental $I(Q)$ data of Figure 2. The coherent scattering domain size is seen to be only about $5 \AA$, with the peaks attenuating rapidly at larger distances. This feature may reflect the glass-like film assembly because the particles are crystalline.

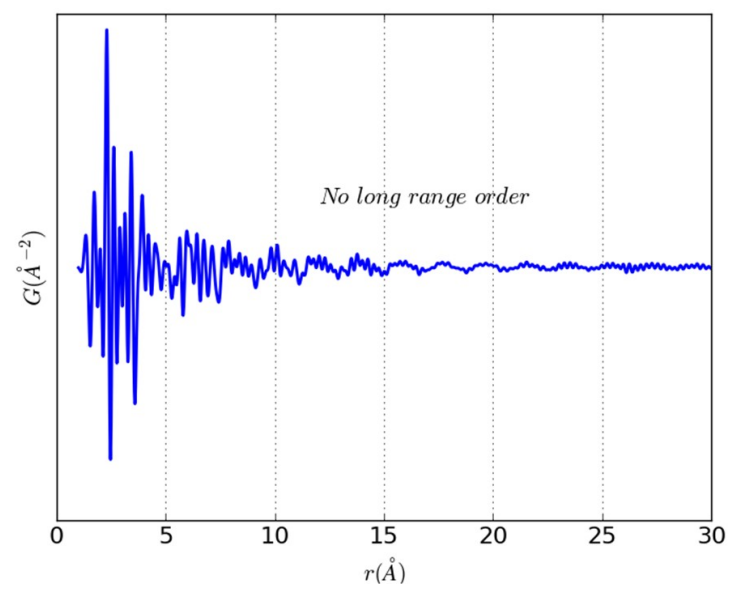

Figure 3. PDF for MnO nanoparticle film sample obtained from the data given in Figure 2.

Figure 4 compares the PDFs for bulk $\mathrm{MnO}$ (cubic rock salt structure) and four different $\mathrm{MnO}_{2}$ polymorphs. $\mathrm{MnO}$ and $\mathrm{MnO}_{2}$ can be distinguished easily by examining the positions of the first two peaks in the PDFs, which are from the $\mathrm{Mn}-\mathrm{O}$ (first peak) and $\mathrm{Mn}-\mathrm{Mn} / \mathrm{O}-\mathrm{O}$ bonds (second peak). The bonding in different $\mathrm{MnO}_{2}$ structures is similar at short range, but differences can be seen in the medium-range bonds. Similarities reflect the presence of $\mathrm{MnO}_{6}$ building blocks that are common to all the $\mathrm{MnO}_{2}$ polymorphs, while differences arise in the details of how these $\mathrm{MnO}_{6}$ octahedra are arranged in space. $\mathrm{MnO}_{6}$ octahedra have been studied earlier by Belli et al. using EXAFS (Extended $\mathrm{X}$-ray absorption fine structure) [46]. 


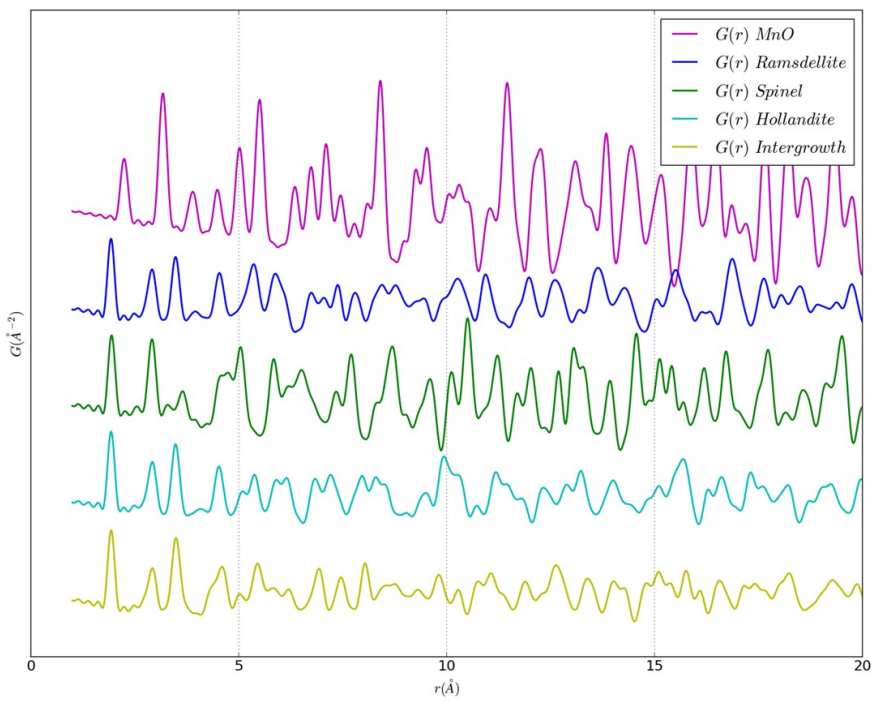

Figure 4. PDFs for bulk $\mathrm{MnO}$ (cubic rock salt structure) and four different $\mathrm{MnO}_{2}$ polymorphs.

Figure 5 shows the computed partial PDFs for bulk MnO. The calculated total PDF shown in Figure 6 is the sum of these partial PDFs. Partial PDFs help in assigning the peaks with their corresponding bonds. Notably, the PDF method can be used to assess the degree of structural coherence in a sample in terms of the experimental PDF. In particular, positions of peaks in the PDF can be related to the distribution of characteristic inter-atomic pair distances in the sample (In our case, the data are seen to be sensitive to only the first few pair distances). A PDF can be easily computed for any model configuration of atoms and thus allows convenient testing and refining of three-dimensional structure models of materials with varying degrees of structural coherence. In this way, periodic and non-periodic models can be evaluated on the same footing and different levels of structural information can be extracted. For crystalline samples, atomic structure can be determined completely, but for less ordered samples, the information obtained is more limited. Figure 5 shows that the experimental data have the highest intensity at the $\mathrm{Mn}-\mathrm{O}$ distance, indicating that we have $\mathrm{MnO}_{6}$ octahedral building blocks or at least distorted/strained octahedral units. The arrangement of the neighboring octahedra is probably quite "random", resulting in destructive signals. A simple fitting to the PDF data has been done in order to extract the lattice constant for the $\mathrm{MnO}$ data. The result of the analysis is shown in Figure 6, and the fitted structure is illustrated in Figure 7 with the lattice constant given by $a=4.56 \AA$. A fit based on a hypothetical 57-atom cluster is discussed in the Supplementary Material.

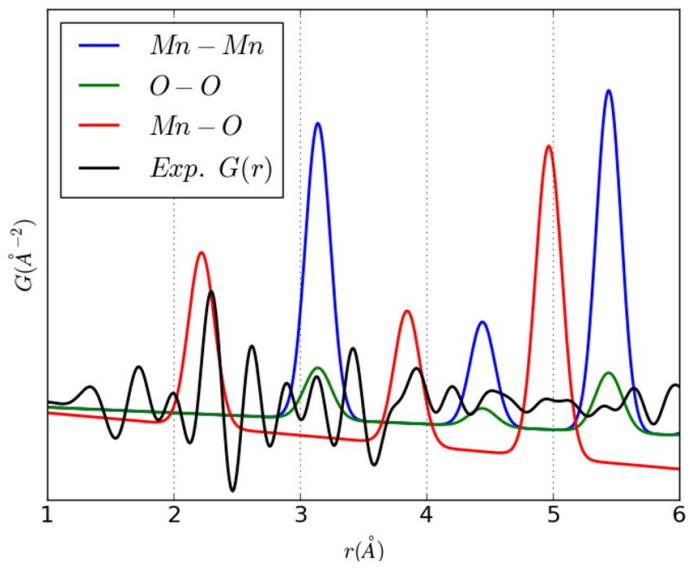

Figure 5. Calculated partial PDFs for bulk MnO. 


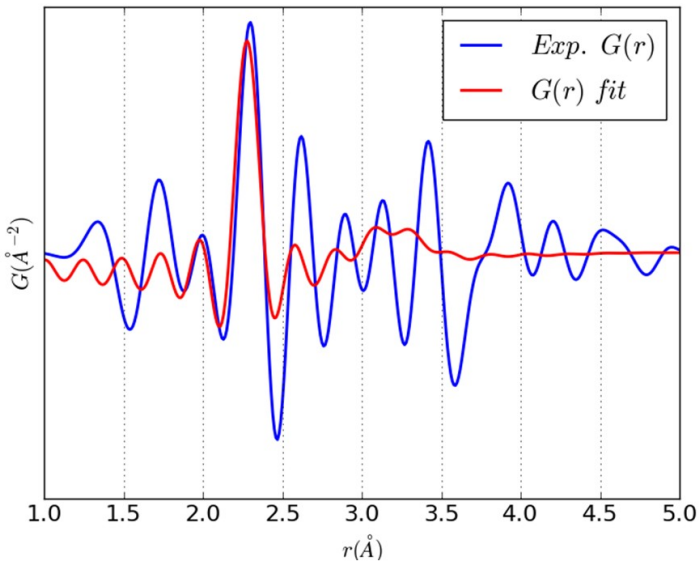

Figure 6. Simple fitting of the data to extract the MnO model with lattice constant $a=4.56 \AA$.

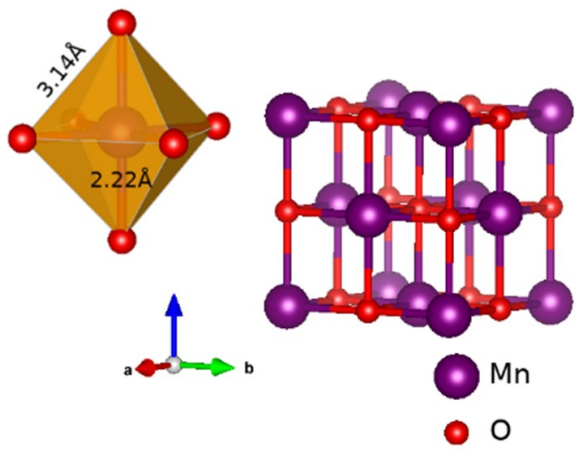

Figure 7. Structural cubic model of $\mathrm{MnO}$.

Our results are quite different from those for synthetic crystalline $\mathrm{MnO}_{2}$ birnessite obtained by Petkov [25], where sharp Bragg peaks are fitted with a model based on a hexagonal lattice rather than the cubic model invoked in our case. Petkov [25] has also shown that very diffuse XRD patterns for bacterial and fungal $\mathrm{MnO}_{\mathrm{x}}$ can be described with monoclinic and triclinic lattices.

\section{Conclusions}

$\mathrm{MnO}$ nanoparticles have attracted much interest due to their potential applications in many fields. In this work, we used HE-XRD at the SPring-8 synchrotron facility to extract important octahedral information, despite the challenging small size of the nanoparticles (about $10 \mathrm{~nm}$ ) and the effect of deposition on the substrate, which results in a glassy thin film as indicated by our X-ray measurements. Our analysis reveals that $\mathrm{Mn}$ atoms are mostly present in an octahedrally coordinated MnO6 form, consistent with a cubic phase with a lattice constant of $a=4.56 \AA$, which is modified by significant glass-like disorder effects. In this way, atomic PDF analysis of HE-XRD data can be used to characterize structural and disorder properties of $\mathrm{MnO}$ nanocrystalline materials used in battery and air purification applications. Clearly, a fundamental understanding of the redox reactions and their relationship with magnetism occurring at the $\mathrm{MnO}_{6}$ sites requires a robust extraction of the octahedron size [47].

Supplementary Materials: The following are available online at http://www.mdpi.com/2410-3896/5/1/19/s1, Figure S1: FFT images for several individual NPs including plane-spacing calculations; Figure S2: Comparison of experimental G(r) with the corresponding simulated results for a hypothetical 57 atom (relaxed) MnO cluster.

Author Contributions: Conceptualization K.P., B.B., and S.K. Experiments Y.V.K., K.O., E.C.-A., J.G., and S.K. Theory and calculations K.P., B.B., and A.B. All authors contributed to the writing of the manuscript. All authors have read and agreed to the published version of the manuscript.

Funding: The work at LUT university was supported by the project Puhdas Ilma from the PROFI 5 support of Academy of Finland (Grant no. 326325). The work at Northeastern University was supported by the US 
Department of Energy (DOE), Oce of Science, Basic Energy Sciences grant number DE-FG02-07ER46352, and benefited from Northeastern University's Advanced Scientific Computation Center (ASCC) and the NERSC supercomputing center through DOE grant number DE-AC02-05CH11231.

Acknowledgments: The HE-XRD experiments were performed with the approval of the Japan Synchrotron Radiation Research Institute (Proposal No.: 2016B1247).

Conflicts of Interest: The authors declare no conflict of interest.

\section{References}

1. Khilari, S.; Pandit, S.; Ghangrekar, M.M.; Das, D.; Pradhan, D. Graphene supported $\alpha-\mathrm{MnO} 2$ nanotubes as a cathode catalyst for improved power generation and wastewater treatment in single-chambered microbial fuel cells. RSC Adv. 2013, 3, 7902. [CrossRef]

2. Post, J. Manganese oxide minerals: Crystal structures and economic and environmental significance. Proc. Natl. Acad. Sci. USA 1999, 96, 3447. [CrossRef] [PubMed]

3. Jin, K.; Chu, A.; Park, J.; Jeong, D.; Jerng, S.E.; Sim, U.; Jeong, H.-Y.; Lee, C.W.; Park, Y.-S.; Yang, K.D.; et al. Partially Oxidized Sub-10 nm MnO Nanocrystals with High Activity for Water Oxidation Catalysis. Sci. Rep. 2015, 5, 10279. [CrossRef] [PubMed]

4. Sinha, A.K.; Suzuki, K.; Takahara, M.; Azuma, H.; Nonaka, T.; Fukumoto, K. Mesostructured Manganese Oxide/Gold Nanoparticle Composites for Extensive Air Purification. Angew. Chem. Int. Ed. 2007, 46, 2891-2894. [CrossRef]

5. Huang, M.; Li, F.; Dong, F.; Zhang, Y.X.; Zhang, L.L. $\mathrm{MnO}_{2}$-based nanostructures for high-performance supercapacitors. J. Mater. Chem. A 2015, 3, 21380-21423. [CrossRef]

6. Hui, X.; Ying, S.M.; Xiaogan, L.; Guoliang, Y.; Chong, C. Porous manganese oxide generated from lithiation/delithiation with improved electrochemical oxidation for supercapacitors. J. Mater. Chem. 2011, 21, 15521-15526. [CrossRef]

7. Tang, N.; Tian, X.; Yang, C.; Pi, Z.; Han, Q. Facile synthesis of $\alpha$-MnO2 nanorods for high-performance alkaline batteries. J. Phys. Chem. Solids 2010, 71, 258-262. [CrossRef]

8. Zhang, Y.; Steiner, J.D.; Uzodinma, J.; Walsh, J.; Zydlewski, B.; Lin, F.; Chen, Y.; Tang, J.; Pradhan, N.; Dai, Q. Thermally synthesized $\mathrm{MnO}$ nanoparticles for magnetic properties and lithium batteries. Mater. Res. Express 2019, 6, 25015. [CrossRef]

9. Tan, Y.; Meng, L.; Peng, Q.; Li, Y. One-dimensional single-crystalline Mn3O4nanostructures with tunable length and magnetic properties of $\mathrm{Mn}_{3} \mathrm{O}_{4}$ nanowires. Chem. Commun. 2011, 47, 1172-1174. [CrossRef]

10. Balamurugan, M.; Venkatesan, G.; Ramachandran, S.; Saravanan, S. Synthesis and Fabrication of Nanomaterials. Bloomsbury India 2015, 1, 31.

11. Cheng, F.F.; Zhao, J.; Song, W.; Li, C.; Ma, H.; Chen, J.; Shen, P. Facile Controlled Synthesis of $\mathrm{MnO}_{2}$ Nanostructures of Novel Shapes and Their Application in Batteries. Inorg. Chem. 2006, 45, 2038-2044. [CrossRef] [PubMed]

12. Tran, D.T.; Ozkaya, D. TEM-based Pair Distribution Function study of interatomic distances in C-supported Pt. J. Phys. Conf. Ser. 2017, 902, 12029. [CrossRef]

13. Guzzinati, G.; Altantzis, T.; Batuk, M.; De Backer, A.; Lumbeeck, G.; Samaee, V.; Batuk, D.; Idrissi, H.; Hadermann, J.; Van Aert, S.; et al. Recent Advances in Transmission Electron Microscopy for Materials Science at the EMAT Lab of the University of Antwerp. Materials 2018, 11, 1304. [CrossRef] [PubMed]

14. Midgley, P.A.; Weyland, M. 3D Electron Microscopy in the Physical Sciences: The Development of Z-Contrast and EFTEM Tomography. Ultramicroscopy 2003, 96, 413-431. [CrossRef]

15. Midgley, P.A.; Dunin-Borkowski, R.E. Electron Tomography and Holography in Materials Science. Nat. Mater. 2009, 8, 271-280. [CrossRef]

16. Ersen, O.; Florea, I.; Hirlimann, C.; Pham-Huu, C. Exploring Nanomaterials with 3D Electron Microscopy. Mater. Today 2015, 18, 395-408. [CrossRef]

17. Möbus, G.; Doole, R.C.; Inkson, B.J. Spectroscopic Electron Tomography. Ultramicroscopy 2003, 96, 433-451. [CrossRef]

18. Liakakos, N.; Gatel, C.; Blon, T.; Altantzis, T.; Lentijo-Mozo, S.; Garcia-Marcelot, C.; Lacroix, L.M.; Respaud, M.; Bals, S.; Van Tendeloo, G. Co-Fe Nanodumbbells: Synthesis, Structure, and Magnetic Properties. Nano Lett. 2014, 14, 2747-2754. [CrossRef] 
19. Goris, B.; Meledina, M.; Turner, S.; Zhong, Z.; Batenburg, K.J.; Bals, S. Three Dimensional Mapping of Fe Dopants in Ceria Nanocrystals Using Direct Spectroscopic Electron Tomography. Ultramicroscopy 2016, 171, 55-62. [CrossRef]

20. Warren, B.E. X-ray Diffraction; Dover Publications Inc.: New York, NY, USA, 1969.

21. Takeshi, E.; Billinge, S.J.L. Underneath the Bragg Peaks: Structural Analysis of Complex Materials; Pergamon Press: Oxford, UK, 2003.

22. Campi, G.; Cappelluti, E.; Proffen, T.; Qiu, X.; Bozin, E.S.; Billinge, S.J.L.; Agrestini, S.; Saini, N.L.; Bianconi, A. Study of temperature dependent atomic correlations in $\mathrm{MgB}_{2}$. Eur. Phys. J. B Condens. Matter Complex Syst. 2006, 52, 15-21. [CrossRef]

23. Proffen, T.; Page, K.L.; Seshadri, R.; Cheetham, A. Pair Distribution Function for Nanoparticle Studies. Los Alamos Sci. 2006, 30, 161.

24. Billinge, S.J.L.; Levin, I. The Problem with Determining Atomic Structure at the Nanoscale. Science 2007, 316, 561-565. [CrossRef] [PubMed]

25. Petkov, V. Nanostructure by high-energy X-ray diffraction. Mater. Today 2008, 11, 28-38. [CrossRef]

26. Norberg, S.T.; Tucker, M.G.; Hull, S. Bond valence sum: A new soft chemical constraint for RMC Profile. J. Appl. Cryst. 2009, 42, 179-184. [CrossRef]

27. Keen, D.A.; Goodwin, A.L. The crystallography of correlated disorder. Nature 2015, 521, 303-309. [CrossRef]

28. Mancini, A.; Malavasi, L. Recent advances in the application of total scattering methods to functional materials. Chem. Commun. 2015, 51, 16592. [CrossRef]

29. Jensen, K.M.Ø.; Blichfeld, A.B.; Bauers, S.R.; Wood, S.R.; Dooryhée, E.; Johnson, D.C.; Iversen, B.B.; Billinge, S.J.L. Demonstration of thin film pair distributionfunction analysis (tfPDF) for the study of localstructure in amorphous and crystalline thin films. IUCrJ 2015, 2, 481-489. [CrossRef]

30. Jensen, K.M.Ø.; Juhas, P.; Tofanelli, M.A.; Heinecke, C.L.; Vaughan, G.; Ackerson, C.J.; Billinge, S.J.L. Polymorphism in magic-sized $\mathrm{Au}_{144}(\mathrm{SR})_{60}$ clusters. Nat. Commun. 2016, 7, 11859. [CrossRef]

31. Dippel, A.-C.; Jensen, K.M.Ø.; Tyrsted, C.; Bremholm, M.; Bøjesen, E.D.; Saha, D.; Birgisson, S.; Christensen, M.; Billinge, S.J.L.; Iversen, B.B. Towards atomistic understanding of polymorphism in the solvothermal synthesis of $\mathrm{ZrO}_{2}$ nanoparticles. Acta Cryst. 2016, 72, 645-650. [CrossRef]

32. Frandsen, B.A.; Brunelli, M.; Page, K.; Uemura, Y.J.; Staunton, J.B.; Billinge, S.J.L. Verification of Anderson Super exchange in MnO via Magnetic Pair Distribution Function Analysis and ab initio Theory. PRL 2016, 197204. [CrossRef]

33. Diaz-Lopez, M.; Freire, M.; Joly, Y.; Colin, C.V.; Fischer, H.E.; Blanc, N.; Boudet, N.; Pralong, V.; Bordet, P. Local Structure and Lithium Diffusion Pathways in $\mathrm{Li}_{4} \mathrm{Mn}_{2} \mathrm{O}_{5}$ High Capacity Cathode Probed by Total Scattering and XANES. Chem. Mater. 2018, 30, 3060-3070. [CrossRef]

34. Christiansen, T.L.; Bøjesen, E.D.; Juelsholt, M.; Etheridge, J.; Jensen, K.M.Ø. Size Induced Structural Changes in Molybdenum Oxide Nanoparticles. ACS Nano 2019, 13, 8725-8735. [CrossRef] [PubMed]

35. Dippel, A.-C.; Roelsgaard, M.; Boettger, U.; Schneller, T.; Gutowskia, U.; Ruett, U. Local atomic structure of thin and ultrathin films via rapid high-energy X-ray total scattering at grazing incidence. IUCrJ 2019, 6, 290-298. [CrossRef] [PubMed]

36. Mathiesen, J.K.; Väli, R.; Härmas, M.; Lust, E.; von Bülow, J.F.; Jensen, K.M.Ø.; Norby, P. Following the in-plane disorder of sodiated hard carbon through operando total scattering. J. Mater. Chem. A 2019, 7, 11709-11717. [CrossRef]

37. Banerjee, S.; Liu, C.-H.; Jensen, K.M.Ø.; Juhás, P.; Lee, J.D.; Tofanelli, M.; Ackerson, C.J.; Murray, C.B.; Billinge, S.J.L. Cluster-mining: An approach for determining core structures of metallic nanoparticles from atomic pair distribution function data. Acta Cryst. 2020, 76, 24-31. [CrossRef]

38. Gallo, J.; Alam, I.S.; Lavdas, I.; Wylezinska-Arridge, M.; Aboagye, E.O.; Long, N.J. RGD-targeted MnO nanoparticles as T1 contrast agents for cancer imaging-The effect of PEG length in vivo. J. Mater. Chem. B 2014, 2, 868-876. [CrossRef]

39. Kohara, S.; Itou, M.; Suzuya, K.; Inamura, Y.; Sakurai, Y.; Ohishi, Y.; Takata, M. Structural studies of disordered materials using high-energy X-ray diffraction from ambient to extreme conditions. J. Phys. Condens. Matter 2007, 19, 506101. [CrossRef]

40. Barbiellini, B.; Platzman, P.M. The healing mechanism for excited moleculesnear metallic surfaces. New J. Phys. 2006, 8, 20. [CrossRef]

41. Lide, D.R. Handbook of Chemistry and Physics, 72nd ed.; CRC Press: Boca Raton, FL, USA, 1991-1992. 
42. King, R.B. Encyclopedia of Inorganic Chemistry; Wiley: Chichester, NY, USA, 1994.

43. Kuriyama, M.; Hosoya, S. X-ray Measurement of Scattering Factors of Manganese and Oxygen Atoms in Manganous Oxide. J. Phys. Soc. Jpn. 1962, 17, 1022-1029. [CrossRef]

44. Juhás, P.; Davis, T.; Farrow, C.L.; Billinge, S.J.L. PDFgetX3: A rapid and highly automatable program for processing powder diffraction data into total scattering pair distribution functions. J. Appl. Cryst. 2013, 46, 560-566. [CrossRef]

45. Juhás, P.; Farrow, C.L.; Yang, X.; Knox, K.R.; Billinge, S.J.L. Complex modeling: A strategy and software program for combining multiple information sources to solve ill posed structure and nanostructure inverse problems. Acta Crystallogr. A 2015, 71, 562-568. [CrossRef] [PubMed]

46. Belli, M.; Scafati, A.; Bianconi, A.; Mobilio, S.; Palladino, L.; Reale, A.; Burattini, E. X-Ray Absorption Near Edge Structures (XANES) In Simple and Complex Mn Compounds. Solid State Commun. 1980, 35, 355-361. [CrossRef]

47. Hafiz, H.; Suzuki, K.; Barbiellini, B.; Orikasa, Y.; Kaprzyk, S.; Tsuji, N.; Yamamoto, K.; Terasaka, A.; Hoshi, K.; Uchimoto, Y.; et al. Identification of ferrimagnetic orbitals preventing spinel degradation by charge ordering in $\mathrm{Li}_{\mathrm{x}} \mathrm{Mn}_{2} \mathrm{O}_{4}$. Phys. Rev. B 2019, 100, 205104. [CrossRef]

(C) 2020 by the authors. Licensee MDPI, Basel, Switzerland. This article is an open access article distributed under the terms and conditions of the Creative Commons Attribution (CC BY) license (http://creativecommons.org/licenses/by/4.0/). 